\title{
Pulmonary Hydatid Disease with Aspergillosis - An Unusual Association in an Immunocompetent Host
}

 \\ Department of 'Pathology and 'Surgery, Dayanand Medical College and Hospital, LUDHIANA, INDIA
}

\begin{abstract}
Echinococcosis is a common cause of pulmonary cavities. Aspergillus fumigatus, a saprophytic fungus, can colonise pulmonary cavities caused by tuberculosis, sarcoidosis, echinococcosis, bronchiectasis and neoplasms. Infection by Aspergillus is often seen in immunosuppressed cases. However, co-infection of Aspergillus with pulmonary echinococcosis is unexpected and very unusual, especially in an immunocompetent patient. We present the case of a 45-year-old immunocompetent male who came with non-resolving pneumonia and fever for 8 months and dyspnoea since 15 days accompanied by recurrent episodes of hemoptysis since 5 days. Chest X Ray and Computed Tomography scan showed a cystic lesion in the middle lobe of the right lung. Middle lobectomy with video-assisted thoracoscopic surgery was performed and histopathology revealed ectocyst of Hydatid cyst which was also colonised by septate fungal hyphae exhibiting acute angled branching, morphologically consistent with Aspergillus. Gomori Methanamine Silver and Periodic Acid Schiff stains highlighted the hyphae of Aspergillus as well as the lamellated membranes of ectocyst and an occasional scolex of Echinococcus. Sections from surrounding lung parenchyma also showed these fungal hyphae within an occasional dilated bronchus. Thus a diagnosis of dual infection of Aspergillosis and Pulmonary Echinococcosis was established. The possibility of dual infection by a saprophytic fungus must be kept in mind while dealing with a case of a cavitary lesion in long-standing and non-resolving pneumonia, even in an immunocompetent patient. Establishing the correct diagnosis of Aspergillosis with Echinococcosis is essential for proper and complete management.
\end{abstract}

Key Words: Pulmonary hydatid cyst, Aspergillosis, Lung

\section{INTRODUCTION}

Hydatid disease or Echinococcosis is a common disease in India caused by the larval form of Echinococcus granulosus. Although any organ can be involved by this parasite, the lung and liver are more frequently affected. Echinococcus multilocularis is the species which commonly involves the lung. Aspergillus fumigatus is a saprophytic fungus which can colonise pulmonary cavities caused by tuberculosis, sarcoidosis, echinococcosis, bronchiectasis and neoplasms (1,2). Infection by Aspergillus is often seen in immunosuppressed cases (3). Co-existence of Aspergillosis and Hydatid disease is a rare occurrence which is seen in immunocompromised patients (4).

We encountered a case of an immunocompetent patient with non-resolving pneumonia and fever who was incidentally found to harbor dual infection with Aspergillus as well as pulmonary hydatid cyst.

\section{CASE REPORT}

A 45-year-old gentleman presented with complaints of breathlessness since 15 days and recurrent episodes of hemoptysis since 5 days. The patient had non-resolving

(Turk Patoloji Derg 2019, 35:166-169)

Received : 20.01.2017 Accepted : 15.03.2017 pneumonia and intermittent fever for the last eight months. He was non-diabetic and did not have any other significant past medical history to suggest immunosuppression. General and systemic examination was unremarkable. $\mathrm{He}$ was non-reactive for HBsAg and HIV. Hemogram was normal and there was no evidence of eosinophilia on peripheral blood examination. No serological examination for Aspergillus or Echinococcus was performed. Pulmonary function tests and liver function tests were normal. Chest $\mathrm{X}$-ray showed a circumscribed cystic lesion in the middle lobe of the right lung. Computed Tomography (CT) scan, done outside, revealed a well-defined peripherally enhancing thick-walled cystic lesion in the middle lobe of the right lung. Ultrasound abdomen did not show any cysts in the liver or any other organ. In view of non-resolving pneumonia and respiratory symptoms, the patient was taken up for surgery. Video-assisted Thoracoscopic Surgery (VATS) middle lobectomy was performed and the cyst was sent for histopathological examination along with part of middle lobe of right lung.

Gross examination revealed a glistening white, thin cyst measuring $4 \times 2.5 \times 2 \mathrm{~cm}$. On opening the cyst, clear fluid

Correspondence: Ruchita TYAGI

Department of Pathology, Dayanand Medical College and Hospital, LUDHIANA, INDIA

E-mail: ruchitatyagi@gmail.com Phone: +919876589911 
was drained out. A part of lung measuring $8 \times 5 \times 2.5 \mathrm{~cm}$ was also received separately in the same container. Cut section of the lung tissue was spongy with hemorrhagic areas. Microscopic examination of cyst wall showed acellular, lamellated eosinophilic membrane - ectocyst with an occasional hooklet highlighted on Gomori's methanamine silver (GMS) stain (Figure 1). Surprisingly, the ectocyst also showed presence of numerous septate hyphae with acute angled branching, conforming with the morphology of Aspergillus (Figure 2). These findings were further confirmed by the GMS and Periodic Acid Schiff (PAS) stains. Thus, a diagnosis of dual infection by Echinococus and Aspergillus was established. The sections from lung tissue showed congestion of alveolar spaces with large areas of necrosis containing interspersed hyphae of Aspergillus, which were also seen to colonise an occasional dilated bronchus. The surrounding lung parenchyma was infiltrated by chronic inflammatory infiltrate along with formation of lymphoid aggregates (Figure 3A-D).

Microbiological examination of cyst fluid or tissue was not done as there was no clinical suspicion of fungal or parasitic infection prior to surgery. The surgically excised tissue was sent for histopathological examination and no material was sent for culture studies.

In view of the dual infection, the patient was started on Itraconazole $400 \mathrm{mg}$ and Albendazole $800 \mathrm{mg}$ orally, daily. As the patient was immunocompetent, the post-operative course was uneventful and there was no dissemination of fungal or parasitic infection. The patient was discharged from hospital within ten days and is on regular follow up.

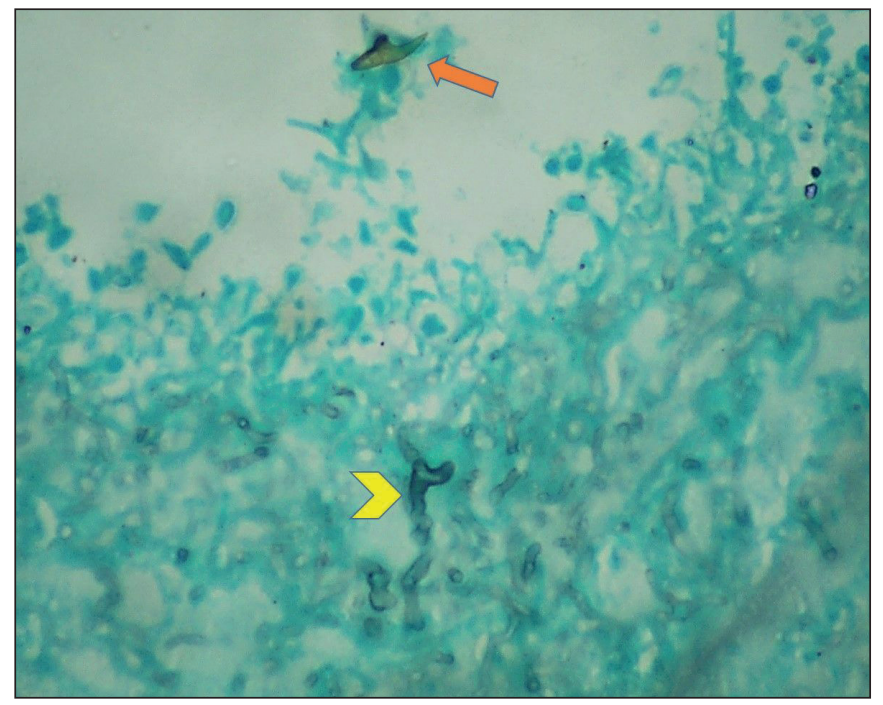

Figure 1: Photomicrograph showing hooklet of Echinococcus (red arrow) along with hyphae of Aspergillus (yellow arrowhead) (GMS; x400).

\section{DISCUSSION}

Aspergillus fumigatus is a saprophytic fungus which can cause allergic pulmonary aspergillosis, aspergilloma, and semi-invasive and invasive aspergillosis in human beings. (1) Rarely, aspergilloma may develop after months or years in an operated hydatid cyst cavity. $(5,6)$ Only a few case reports have described the involvement of an active hydatid cyst cavity by Aspergillus in immunocompetent patients $(1,2,4,7-16)$.

About $60 \%$ of pulmonary hydatid disease has been reported to affect the right lung, as was also seen in our case. While the middle lobe was involved in our case, $50 \%$ to $60 \%$ of pulmonary cases are seen in the lower lobes $(1,7,8,17)$. The clinical presentation of pulmonary echinococcosis depends on the site, size of the cyst and whether the cyst has ruptured or is intact. Intact cyst may be an incidental finding or may cause cough, dyspnoea and chest pain due to compression of surrounding structures. A cyst may rupture into a bronchus, pleura or a vessel, causing hemoptysis. The patient may expectorate cyst contents, have recurrent episodes of hemoptysis, productive cough, fever or even anaphylactic shock due to aspiration of cyst contents in such cases $(1,2,7,9,18)$. In our case, similar presenting features were seen in the form of fever and non-resolving pneumonia for eight months, dyspnoea since 15 days and recurrent episodes of hemoptysis since 5 days. Eosinophilia can occur in $40 \%$ of cases. Serological tests for Echinococcus include Casoni's intradermal test, hemagglutination tests, flocculation tests, precipitin reaction, complement fixation test. Indirect fluorescent antibody test and immune-

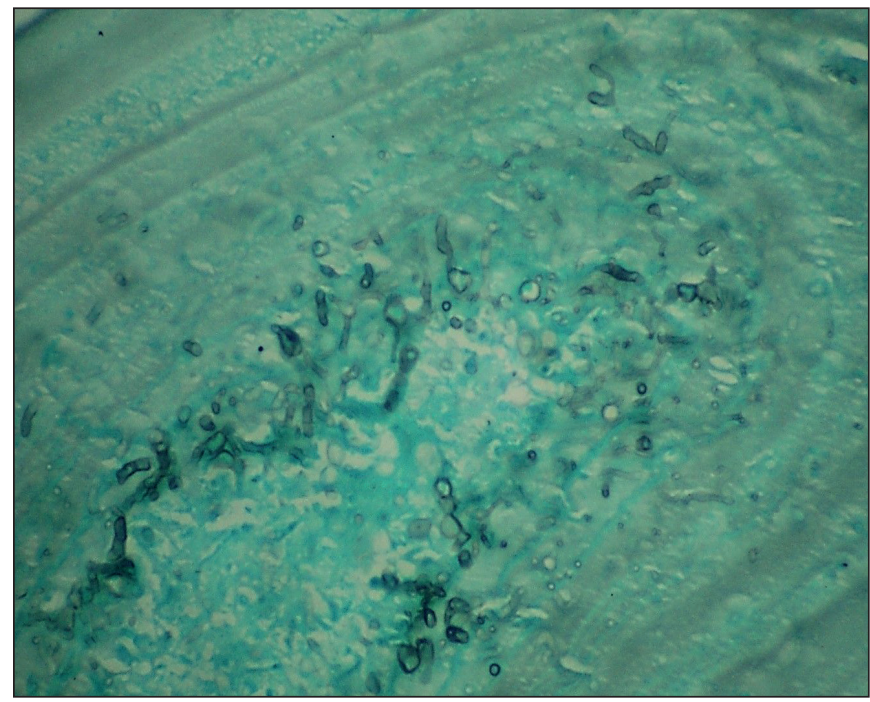

Figure 2: GMS stain highlighting acellular lamellated ectocyst with fungal hyphae (GMS; x400). 
electrophoresis have also been used (18). However, there was no eosinophilia in peripheral blood examination in our patient. Aspergillus galactomannan Antigen (AGA) levels may help in detecting invasive aspergillosis infection (13). However, no such serological tests were performed in this case.

Other organisms that are known to cause secondary infection with hydatid cyst include Escherichia coli and viridans group streptococci in hepatic cysts and Aspergillus fumigatus in pulmonary cysts (4). Fungal colonization may occur because of immunosuppression, structural defects in lungs, prior intervention or cyst rupture $(2,9,10)$. Aspergillus may have a propensity to colonise cysts close to the hilum as obliteration of the cavity does not occur in these cases (10). The large size of the hydatid cyst may predispose to secondary infection, which may even result in the formation of an abscess, causing purulent sputum $(6,18)$. However, in many instances, fungal colonization has been observed even in the absence of the above mentioned predisposing conditions (15).

Surgical resection is the treatment of choice for pulmonary echinococcosis. If Aspergillus is limited to the hydatid cyst cavity, then resection is curative for both the infections. In our case, aspergillus was also present in the lung parenchyma and bronchi. In such cases, oral antifungal treatment is also required. In unresectable, recurrent or inoperable echinococcosis, oral anti-helminthic therapy is warranted $(13,18)$. As our patient was immunocompetent, there was no dissemination of Aspergillus and the postoperative stay was uneventful.

In conclusion, we hereby report an unusual occurrence of non-resolving pneumonia caused by dual infection comprising of Aspergillosis in a case of pulmonary hydatid cyst, detected on histopathological examination. Even in an immunocompetent patient, the possibility of


Figure 3: A) Section from lung showing areas of necrosis surrounded by chronic inflammatory infiltrate (H\&E; x100). B) Septate fungal hyphae of Aspergillus with acute angled branching in necrotic areas (H\&E; $\mathrm{x} 400)$. C) Hyphae of Aspergillus colonising ectocyst (H\&E; x400). D) PAS stain highlighting fungal hyphae of Aspergillus against background of ectocyst of Echinococcus (PAS; x100) . 
secondary infection of a hydatid cyst cavity by saprophytic fungus should be kept in mind for better and complete management.

\section{REFERENCES}

1. Dhal I, Sahoo TP, Behera SS, Panigrahy SK, Nayak R, Ali SI. Pulmonary hydatid cyst with Aspergillous fungal co-infection: A rare case report and review of literature. Int J Dent Med Res. 2015;1:97-9.

2. Kocer NE, Kibar Y, Guldur ME, Deniz H, Bakir K. A retrospective study on the coexistence of hydatid cyst and aspergillosis. Int $\mathrm{J}$ Infect Dis. 2008;12:248-51.

3. Soubani AO, Chandrasekhar $\mathrm{PH}$. The clinical spectrum of pulmonary aspergillosis. Chest. 2002;121:1988-99.

4. Manzoor MU, Faruqui ZS, Ahmed Q, Uddin N, Khan A. Aspergilloma complicating newly diagnosed pulmonary echinococcal (hydatid) cyst: A rare recurrence. Br J Radiol. 2008;81:279-81.

5. Ulasan NG, Dural K, Yildirim E, Ozisik K, Sakinci U. An intracavitary aspergilloma after echinococcal cystectomy. J Cardiovasc Surg (Torino). 2003;44:289-90.

6. Aydemir B, Aydemir C, Okay T, Celik M, Dogusoy I. An aspergilloma in echinococcal cyst cavity. Thorac Cardiovasc Surg. 2006;54:353-5.

7. Pan JB, Hou YH, Yin PZ. A case report of hydatid cysts containing aspergillus. J Thorac Dis. 2013;5:E25-E27.

8. Agarwal S, Bohara S, Thakran A, Arora P, Singh R, Agarwal PN. Pulmonary hydatid disease with coexistent aspergillosis: An incidental finding. Indian J Med Microbiol. 2013;31:85-6.
9. Mahapatra S, Das R, Mohapatra C, Rath J, Behera S. Aspergillosis in Hydatid cyst - a rare coexistence. Virol Mycol. 2012;1:106.

10. Agrawal M, Uppin MS, Manasa PL, Uppin SG, Chakravarty MP, Mishra RC, Challa S. Aspegillus colonization in hydatid cyst: Addition of a case. Lung India. 2013;30:335-7.

11. Bal A, Bagai M, Mohan H, Dalal U. Aspergilloma in a pulmonary hydatid cyst: A case report. Mycoses. 2008;5:357-9.

12. Gupta N, Arora J, Nijhawan R, Aggarwal R, Lal A. Aspergillosis with pulmonary echinococosis. Cytojournal. 2006;3:7.

13. Chatterjee A, Goswami HM, Dhotre SV, Dharsandia MV, Raval AA, Vegad MM. A retrospective study on coexistence of pulmonary hydatid cyst and aspergillosis. Int J Microbiol Res. 2012;4:279-81.

14. Vaideeswar P, Vyas M, Katewa A, Bhaskar M. Piggyback mycosis: Pulmonary hydatid cyst with a mycotic co-infection. Mycoses. 2010;53:265-8.

15. M'saad S, Ayedi L, Abdennader M, Bahloul N, Hentati A, Dabbech C, et al. Aspergilloma in a hydatid cavity: A case report. Respir Med CME. 2010;3:29-32.

16. García MB, Lledías JP, Pérez IG, Tirado VV, Pardo LF, Bellvís LM, Varela G, Sánchez MC. Primary super-infection of hydatid cystclinical setting and microbiology in 37 Cases. Am J Trop Med Hyg. 2010;82:376-8.

17. Bhatia G. Echinococcus. Semin Resp Infec. 1997;12:171-86.

18. Mahmood N, Azam H, Ali MI, Khan MA. Pulmonary hydatid cyst with complicating Aspergillus infection presenting as a refractory lung abscess. Clin Med Insights Case Rep. 2011;4:63-8. 